\title{
O PERFIL DA MULHER QUE SE SUBMETE A MAMOGRAFIA EM GOIÂNIA-UMA CONTRIBUIÇÃO A “BASES PARA UM PROGRAMA DE DETECÇÃO PRECOCE DO CÂNCER DE MAMA"
}

\author{
Eduardo Rodrigues Godinho' ${ }^{1}$, Hilton Augusto Koch²
}

Resumo Foi estudado o perfil de 2.000 mulheres que se submeteram a mamografia em um hospital público e uma clínica privada de Goiânia, GO. Os dados foram coletados em entrevista. As mulheres tinham, em média, 49 anos de idade, a maioria procedia da própria cidade, 11,5\% nunca tinham amamentado e dois terços tinham amamentado por mais de seis meses. Terapia de reposição hormonal era utilizada por mais de $20 \%$, e um quinto destas não havia se submetido a mamografia previamente. História de câncer de mama familiar foi relatada por $13,3 \%$ das examinadas no hospital público e por $7,5 \%$ das examinadas na instituição privada. Destas, $28,67 \%$ e 33,33\%, respectivamente, não tinham mamografia preliminar. Rastreamento do câncer mamário foi a maior motivação para o exame. Grande parte das entrevistadas não havia se submetido a mamografia anteriormente, por falta de solicitação médica ou por a considerarem desnecessária na época do pedido.

Unitermos: Mamografia. Câncer de mama. Rastreamento.

Abstract The profile of the women who submit to mammography in the city of Goiânia, Brazil - a contribution to the "Basis for early breast cancer detection program".

We studied the profile of 2,000 women who were submitted to mammography studies at a public hospital and at a private clinic in the city of Goiânia, GO, Brazil. Data were collected through interviews. The mean age of the women was 49 years. Most women resided in the city, $11.5 \%$ had not breastfed whereas two thirds had breastfed for more than 6 months. Hormone-replacement therapy was used by over $20 \%$ of the women, of which one fifth had never been submitted to a mammography study. Family history of breast cancer was reported in $13.3 \%$ of the women examined at the public hospital and in $7.5 \%$ of the women examined at the private clinic. Of these, $28.67 \%$ and $33.33 \%$, respectively, had never been submitted to a mammography study before. Screening for cancer was the main cause that motivated the exam. A great number of the interview ed women had not been submitted to a mammography study due to the lack of a medical request, or simply because they considered it unnecessary at the time of request.

Key words: Mammography. Breast cancer. Screening.

\section{INTRODUÇÃO}

Ocâncer de mama, segundo estimativas do Instituto Nacional do Câncer (INCA), deverá vitimar cerca de 31.590 mulheres no Brasil no ano de 2001. O número de óbitos previsto é de aproximadamente 8.670. Estas estimativas representam uma taxa bruta de incidência de 36,47/100.000 e taxa bruta de mortalidade de 9,99/100.000. Entre 1979 e 1998 a taxa bruta de mortalidade era de cerca de 5,77/100.000, obser-

1. Pós-graduando (Mestrado) em Radiologia da Faculdade de Medicina da Universidade Federal do Rio de J aneiro (UFRJ ), Rio de J aneiro, RJ

2. Professor Titular de Radiologia da UFRJ, Coordenador da linha de pesquisa "Bases para um programa de deteç̧ão precoce do câncer de mama por meio da mamografia", do Curso de Pós-graduação em Radiologia da UFRJ .

Endereço para correspondência: Dr. Eduardo Rodrigues Godinho. Rua 5, no 784, Centro. Goiânia, G0, 74055-290. E-mail: eduardorgodinho@ig.com.br

Recebido para publicação em 7/11/2001. Aceito, após revisão, em 3/12/2001. vando-se, portanto, um aumento de aproximadamente $68 \%$ dessa taxa ${ }^{(1,2)}$.

Elevação na incidência do câncer de mama tem sido relatada mundialmente $\mathrm{e}$ não é proveniente apenas da maior disponibilidade de métodos diagnósticos ${ }^{(3)}$. Estudos apontam que no período 1975-2000 o número de casos novos, em mulheres idosas, deve aumentar $15 \%$ em países desenvolvidos e $63 \%$ em países em desenvolvimento $^{(4)}$. No Brasil, a taxa de mortalidade por esta modalidade de câncer saltou de 5,77/100.000 em 1979 para 9,70/100.000 em $1998^{(2)}$.

Algumas alterações nas características da população feminina têm sido responsabilizadas pelo incremento na incidência do câncer de mama. No Brasil, o processo de urbanização pelo qual tem passado o país (fato ocorrido há mais tempo nas nações desenvolvidas), sobretudo a partir da década de 70 , torna maior a exposição das mulheres a fatores que elevam o risco para o desenvolvimento do câncer de mama. Dentre esses fatores, a primeira gravidez tardia e a obesidade. O aumento da expectativa de vida da população brasileira também tem exercido influência, já que a incidência do câncer de mama cresce com o progredir da idade ${ }^{(5-8)}$. O lapso temporal existente na mudança dessas características da população feminina deve representar a explicação para as estimativas de maior número de casos novos nas nações em desenvolvimento.

\section{Prevenção do câncer de mama}

A questão da prevenção do câncer de mama é bastante complexa, em virtude da multiplicidade de fatores envolvidos, tornando difícil o controle.

História familiar, presença do gene BRCA $^{(9-12)}$, hormônios endógenos e exógenos, fatores sócio-demográficos, tipo de 
dieta e estilo de vida, todos são co-participantes, porém a capacidade de intervenção nesses fatores é limitada ${ }^{(\mathbf{1 0 , 1 2})}$.

A prevenção do câncer de mama pode ser primária ou secundária. O papel da prevenção primária é o de modificar ou eliminar fatores de risco para este câncer. $\mathrm{Na}$ prevenção secundária enquadram-se o diagnóstico e tratamento dos cânceres pre$\operatorname{coces}^{(\mathbf{1 0})}$. Nesta abordagem está inserida a mamografia.

\section{A mamografia}

A mamografia é apontada como o método mais sensível, na atualidade, para detecção do câncer de mama em estádio préinvasivo $^{(13-15)}$, ocasião em que o índice de cura tem alcançado até $95 \%{ }^{(\mathbf{1 3})}$. Sua sensibilidade é alta, mas se reduz progressivamente, proporcionalmente ao intervalo de tempo de acompanhamento após sua realização. A sensibilidade também é afetada pela composição das mamas, sendo menor nas mamas densas, e pela história familiar de câncer de mama, provavelmente em conseqüência do rápido crescimento do tumor ${ }^{(16)}$.

A mamografia está indicada para dois grupos distintos: 1) mulheres assintomáticas, sendo utilizada como exame de rastreamento do câncer de mama; 2) mulheres sintomáticas, nas quais os achados clínicos levam à suspeita de câncer de mama.

Muito se questionou quanto à validade da mamografia em mulheres abaixo dos 50 anos de idade, alegando-se que seu uso em programas de rastreamento não induz à redução da mortalidade. A propensão atual é a aceitação das evidências de que esta prática produz diminuição significativa da morbidade e da mortalidade em todos os grupos etários acima de $40 \operatorname{anos}^{(\mathbf{1 7 - 2 2})}$

Quanto ao intervalo de tempo entre os exames mamográficos, trabalhos têm demonstrado que a sensibilidade da mamografia é a mesma para mulheres entre $40 \mathrm{e}$ 49 anos examinadas anualmente e naquelas com mais de 50 anos avaliadas a cada dois anos ${ }^{(\mathbf{1 6})}$.

Michaelson et al. ${ }^{(23)}$, utilizando método de simulação computadorizada, estimaram que o rastreamento a cada dois anos produziria redução de cerca $22 \%$ na taxa de doença metastática a distância, um intervalo de um ano resultaria em $51 \%$ de redu- ção, e um intervalo de seis meses minoraria em $80 \%$ esta taxa.

A discussão do câncer radioinduzido também é cogitada. Ferreira et al. ${ }^{\mathbf{( 2 4 )}}$, em trabalho buscando estabelecer riscos e benefícios do uso da mamografia, concluíram serem claros os benefícios em todas as faixas etárias a partir dos 40 anos, e que com eficiência do rastreamento mamográfico superior a $40 \%$ o uso da mamografia de alta resolução é plenamente justificável.

\section{Programa de detecção precoce do câncer de mama pela mamografia}

O Brasil não possui um programa oficial de rastreamento do câncer de mama. O Colégio Brasileiro de Radiologia (CBR), juntamente com a Federação Brasileira de Ginecologia e Obstetrícia e Sociedade Brasileira de Mastologia formaram uma comissão conjunta que tem procurado nortear o uso deste método diagnóstico no país. Esta comissão organizou a edição de um livro, "Mamografia atual", escrito por autoridades brasileiras no assunto. Nele encontrase referendada a recomendação da American Cancer Society (ACS) quanto ao uso da mamografia de rastreamento: 1) mulheres entre 35 e 40 anos de idade - realização da mamografia de base, a qual servirá de comparação às próximas; 2) mulheres entre 40 e 49 anos de idade - a mamografia deverá ser realizada a intervalos de um ou dois anos, estando esta freqüência dependente dos fatores de risco individuais; 3) mulheres com 50 anos de idade ou mais - devem submeter-se a mamografias, com intervalos de um ano; 4) mulheres com história pessoal ou familiar de câncer de mama-devem orientar-se junto ao seu médico quanto à necessidade de iniciar o rastreamento antes dos $40 \operatorname{anos}^{(25)}$.

\section{Justificativa}

A literatura consultada revela escassez de trabalhos buscando delinear o perfil da mulher que se submete ao exame mamográfico. São localizados artigos abordando aspectos isolados, tais como faixa etária das mulheres examinadas, motivações clínicas para a realização da mamografia e o grau de conhecimento do método pela populaçãofeminina.

Pelo levantamento bibliográfico realizado verifica-se não existirem informações disponíveis que permitam reconhecer as características da mulher que se submete ao exame mamográfico. Quais seriam as respostas às seguintes perguntas: Com que idade tem chegado a mulher para a primeira mamografia? Qual a freqüência do uso da terapia de reposição hormonal (TRH), e que cuidados especiais têm recebido as mulheres em hormonioterapia para prevenção do câncer de mama pela mamografia? $\mathrm{O}$ que tem mais comumente motivado a solicitação deste exame pelo médico assistente? Que porcentagem das mulheres possui mamografia anterior? Quando não têm, e já deveriam ter, onde está a falha do processo: no médico assistente, na estrutura do sistema de saúde, na desinformação da mulher? Qual o intervalo médio de tempo observado entre os exames mamográficos? Como tem sido conduzido o rastreamento mamográfico das mulheres com fatores de risco para desenvolvimento de câncer de mama? Qual a procedência da mulher que realiza avaliação mamográfica: da própria cidade, do interior ou de outro estado?

A busca de respostas para estas questões é fundamental para a estruturação de um programa de detecção precoce do câncer de mama. Supostamente, a aderência ao programa será maior se este for traçado de maneira estratégica, antecipando-se às adversidades que, no momento, podem estar obscurecidas pela falta de estudos específicos na área.

\section{Objetivos}

Traçar o perfil da mulher que se submete a mamografia em Goiânia, GO, procurando utilizar os resultados na análise das eventuais falhas no processo de rastreamento do câncer mamário.

\section{CASUÚSTICA E MÉTODO}

Foi realizado estudo prospectivo, por meio de entrevista, de 2.000 mulheres que, por demanda, se dirigiram a um dos centros participantes da pesquisa para realização de exame de mamografia, no período compreendido entre outubro de 2000 e julho de 2001.

Cada mulher foi incluída no estudo apenas na ocasião da realização do primeiro exame mamográfico, sendo excluída na eventualidade de retorno por qualquer 
motivo. Participaram da pesquisa dois serviços de radiologia, instalados na cidade de Goiânia, GO: a) hospital público universitário (Hospital das Clínicas da Universidade Federal de Goiás); b) clínica privada (Núcleo Radiológico).

Foram consideradas fora de TRH as mulheres que não estavam em hormonioterapia há seis meses ou mais. Foram reconhecidos como casos de câncer de mama na família apenas aqueles com parentesco em primeiro grau.

As mamografias anteriormente realizadas foram consideradas numericamente até o número de 3 e, a partir de 4, discriminadas como 4 ou mais. Buscou-se ainda determinar o tempo decorrido desde o último exame mamográfico.

Foram enquadradas na categoria "Médico nunca pediu" as mulheres que se consultavam regular ou ocasionalmente, mas a mamografia não havia sido solicitada.

Foi criado um banco de dados com base no sistema Access 97. Os cálculos das médias e das porcentagens foram realizados no Excel 97.

\section{RESULTADOS}

A avaliação por faixa etária revelou idade média de 49,17 anos no hospital público e também de 49,17 anos na clínica privada. No subgrupo etário de 35-49 anos, a média de idade em que a mulher realizou a mamografia pela primeira vez no hospital público foi de 43,9 anos, e no serviço particular a idade média foi de 41,8 anos. Este grupo etário representou 57,2\% das mulheres examinadas no primeiro centro e $52,7 \%$ no segundo.

Constatou-se que $62,3 \%$ das mulheres que se submeteram a mamografia no hospital público eram procedentes de Goiânia. As do interior do estado de Goiás e de outros estados corresponderam a $34,8 \%$ e $2,9 \%$, respectivamente. Na clínica privada, estes números foram, na mesma seqüência, $78 \%, 18 \%$ e $4 \%$.

No hospital público, 22,1\% $(\mathrm{n}=221)$ das mulheres estavam em TRH, e na clínica privada, $27,5 \%(n=275)$. Destas mulheres em TRH, 15,38\% $(n=34)$ das que se dirigiram ao hospital público e 23,27\% (n =64) das que se dirigiram à clínica privada não possuíam mamografia anterior.
A avaliação da história familiar de câncer de mama, a relação com o uso de TRH e a avaliação mamográfica prévia estão detalhadas na Tabela 1. As indicações clínicas que motivaram os exames em ambos os centros estão mostradas na Tabela 2 . História de procedimento intervencionista mamário está detalhada na Tabela 3.

As mulheres com idade superior a 40 anos representaram 94\% $(n=940)$ das examinadas no serviço público e $82,8 \%$ das examinadas no serviço particular $(\mathrm{n}=828)$. Na primeira instituição, 33,08\% $(n=311)$ não tinham realizado mamografia anteriormente, e na segunda, 43,11\% $(\mathrm{n}=357)$.

A distribuição etária das mulheres submetidas a mamografia em ambos os centros é apresentada na Figura 1. Os motivos apontados pelas mulheres para não terem realizado mamografia estão mostrados na
Figura 2. Cerca de $33 \%$ das mulheres entrevistadas na clínica particular e $43 \%$ no hospital público não tinham mamografia anterior. Houve queda no número de examinadas com o avançar da idade (Figura 3 ).

\section{DISCUSSÃO}

O estudo foi realizado em Goiânia, GO. O município tem 1.090.581 habitantes, dos quais 570.903 do sexo feminino, de acordo com o censo 2000 do IBGE ${ }^{(26)}$. A cidade funciona como pólo de assistência médica na Região Centro-Oeste, contando com 73 hospitais, cinco postos de saúde, 45 centros de saúde e 893 consultórios médicos ${ }^{(27)}$.

Não houve afluência significativa de mulheres de outras unidades da federação para estudo mamográfico em Goiânia; no

Tabela 1 Porcentagem de mulheres com história familiar de câncer de mama e o uso da mamografia nessas mulheres.

\begin{tabular}{|l|c|c|}
\hline \multirow{2}{*}{} & \multicolumn{2}{|c|}{ Mulheres com parentes em $1^{\circ}$ grau com câncer de mama } \\
\cline { 2 - 3 } & Hospital público & Clínica privada \\
\hline Total & $13,3 \%(n=133)$ & $7,5 \%(\mathrm{n}=75)$ \\
Sem mamografia prévia & $28,67 \%(\mathrm{n}=38)$ & $33,33 \%(\mathrm{n}=25)$ \\
& Idade média $=47$ anos & Idade média $=45$ anos \\
Em TRH sem mamografia prévia & $21,05 \%(\mathrm{n}=8)$ & $4 \%(\mathrm{n}=1)$ \\
\hline
\end{tabular}

Tabela 2 Indicações clínicas que motivaram a realização de mamografia, subdividindo-se as mulheres em grupos de idade menor e maior que 35 anos, de acordo com o centro onde foi realizado o exame.

\begin{tabular}{|c|c|c|c|c|c|c|c|c|}
\hline & \multicolumn{4}{|c|}{ Clínica privada } & \multicolumn{4}{|c|}{ Hospital público } \\
\hline & \multicolumn{2}{|c|}{$\begin{array}{c}\text { Menos de } 35 \text { anos } \\
(n=43)\end{array}$} & \multicolumn{2}{|c|}{$\begin{array}{l}\text { Mais de } 35 \text { anos } \\
(\mathrm{n}=957)\end{array}$} & \multicolumn{2}{|c|}{$\begin{array}{c}\text { Menos de } 35 \text { anos } \\
(n=6)\end{array}$} & \multicolumn{2}{|c|}{$\begin{array}{c}\text { Mais de } 35 \text { anos } \\
\quad(n=994)\end{array}$} \\
\hline & $\mathrm{n}$ & $\%$ & $\mathrm{n}$ & $\%$ & $\mathrm{n}$ & $\%$ & $\mathrm{n}$ & $\%$ \\
\hline Rotina & 7 & 16,28 & 676 & 70,2 & 1 & 5,88 & 613 & 62,36 \\
\hline Nódulo & 11 & 25,58 & 55 & 6 & 6 & 35,29 & 126 & 12,81 \\
\hline Mastalgia & 19 & 44,18 & 149 & 16 & 6 & 35,29 & 147 & 14,95 \\
\hline Pré-operatório & 6 & 13,95 & 6 & 0,46 & 3 & 17,64 & 40 & 4,06 \\
\hline Outros & 0 & 0 & 71 & 7,3 & 1 & 5,88 & 57 & 5,79 \\
\hline
\end{tabular}

Tabela 3 Procedimentos intervencionistas mamários mais freqüentes, analisados isoladamente e associados ao uso da mamografia, anteriormente à intervenção (excluindo-se a categoria "outros").

\begin{tabular}{|c|c|c|c|c|c|c|c|c|}
\hline & \multicolumn{4}{|c|}{ Clínica privada $(n=70)$} & \multicolumn{4}{|c|}{ Hospital público $(n=138$ ) } \\
\hline & \multirow[t]{2}{*}{$\mathrm{n}$} & \multirow[t]{2}{*}{$\%$} & \multicolumn{2}{|c|}{$\begin{array}{l}\text { Sem mamo- } \\
\text { grafia prévia }\end{array}$} & \multirow[t]{2}{*}{$\mathrm{n}$} & \multirow[t]{2}{*}{$\%$} & \multicolumn{2}{|c|}{$\begin{array}{l}\text { Sem mamo- } \\
\text { grafia prévia }\end{array}$} \\
\hline & & & $n$ & $\%$ & & & $\mathrm{n}$ & $\%$ \\
\hline Retirada de nódulo & 22 & 31,42 & 6 & 27,27 & 85 & 61,3 & 5 & 5,88 \\
\hline Punção & 20 & 28,57 & 1 & 5,00 & 14 & 6,4 & 0 & - \\
\hline Plástica & 21 & 30,00 & 8 & 38,09 & 13 & 9,7 & 3 & 23,08 \\
\hline Mastectomia & 4 & 5,71 & 2 & 50,00 & 22 & 12,9 & 0 & - \\
\hline Prótese & 3 & 4,28 & 1 & 33,33 & 1 & 0,72 & 0 & 0 \\
\hline Retirada de microcalcificações & 0 & 0 & 0 & 0 & 3 & 2,17 & 0 & 0 \\
\hline
\end{tabular}




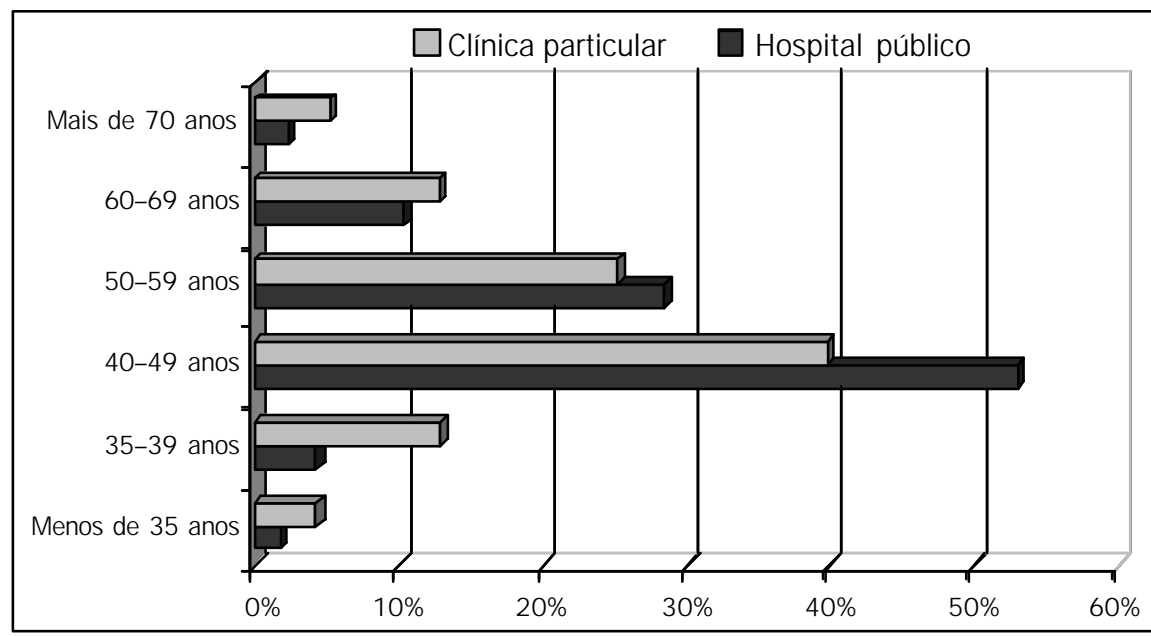

Figura 1. Distribuição etária das mulheres que se submeteram a mamografia no hospital público e na clínica privada.

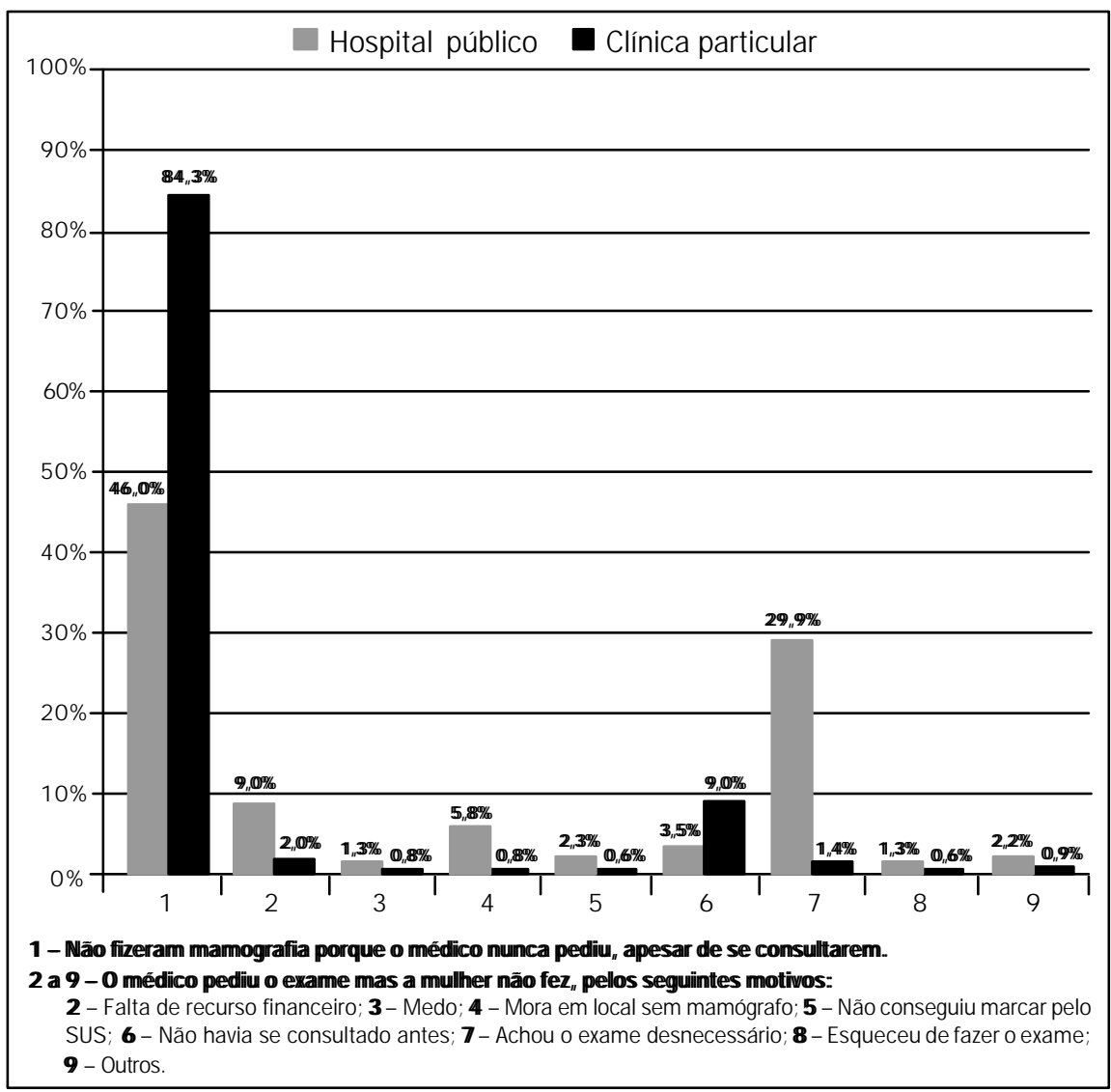

Figura 2. Principais justificativas referidas pelas mulheres com mais de 40 anos de idade que nunca realizaram mamografia.

entanto, cerca de um terço das examinadas no serviço público era procedente do interior do estado. Este aspecto deve ser considerado cuidadosamente no planejamento de um programa de rastreamento do câncer de mama em âmbito nacional, já que com a municipalização da saúde as prefei- turas dos grandes centros urbanos podem procurar privilegiar as mulheres residentes em suas áreas de administração. Outro aspecto já levantado por Koch et al. ${ }^{(28)}$ é a insuficiência de equipamentos disponíveis no país, o que se agravaria com a procura pelo exame nas cidades de maior porte.
Durante o período de coleta dos dados no hospital público, o tempo médio de espera pela mamografia era de dois a três meses. Mesmo assim, interessantemente, as mulheres não alegaram este motivo como limitador à sua realização.

A idade média de comparecimento para o primeiro estudo mamográfico foi de 49 anos, demonstrando que o rastreamento do câncer mamário nesta faixa etária não está ocorrendo de forma efetiva. Cerca de $20 \%$ dos casos de câncer de mama ocorrem na quinta década de vida ${ }^{(29)}$, sendo que a acurácia da mamografia entre os 35 e 49 anos está em torno de $96 \%$, com sensibilidade de $83 \%$ e especificidade de $97 \%{ }^{(30)}$.

Apenas 4,3\% das examinadas no hospital público estão compreendidas entre 35 e 39 anos de idade, e na instituição particular, $13 \%$, dando indícios de que a mamografia de base não tem sido adotada pelos médicos, ao menos na instituição pública, ou não venha sendo valorizada pela população feminina desta idade, que pode estar deixando de comparecer para realizá-la. Pelo censo de 1996 do IBGE ${ }^{(\mathbf{2 6})}$, este grupo etário compreendia $24 \%$ da população feminina da cidade.

Apesar da realização tardia do primeiro exame, existe grande concentração destes entre os 40 e 49 anos (39,8\% na clínica particular e $52,9 \%$ no hospital público), números que corroboram aqueles citados por Marconato e Boff ${ }^{(29)}$ no Rio Grande do Sul. As entrevistadas com idade superior a 40 anos sem mamografia anterior foi alto. Estas proporções elevadas são semelhantes às encontradas por Vieira $\mathrm{Koch}^{(\mathbf{3 1})}$,em pesquisa conduzida na Santa Casa de Misericórdia do Rio de Janeiro, porém bem inferiores aos $88 \%$ relatados por Lamaset $a l{ }^{(32)}$, em estudo realizado em serviço privado do Distrito Federal, e por Baségio e $\operatorname{Koch}^{(6)}$, no Rio Grande do Sul. Dentre os motivos geradores desses resultados, uma parcela importante é oriunda da ausência de solicitação da mamografia pelo médico assistente. A falta de solicitação médica como entrave ao processo de rastreamento do câncer de mama também foi encontrado no trabalho de Vieira e $\operatorname{Koch}^{(31)}$.

Um terço das examinadas no serviço público disse não ter realizado mamografia anteriormente, apesar de solicitada, por julgar o exame desnecessário na ocasião. 


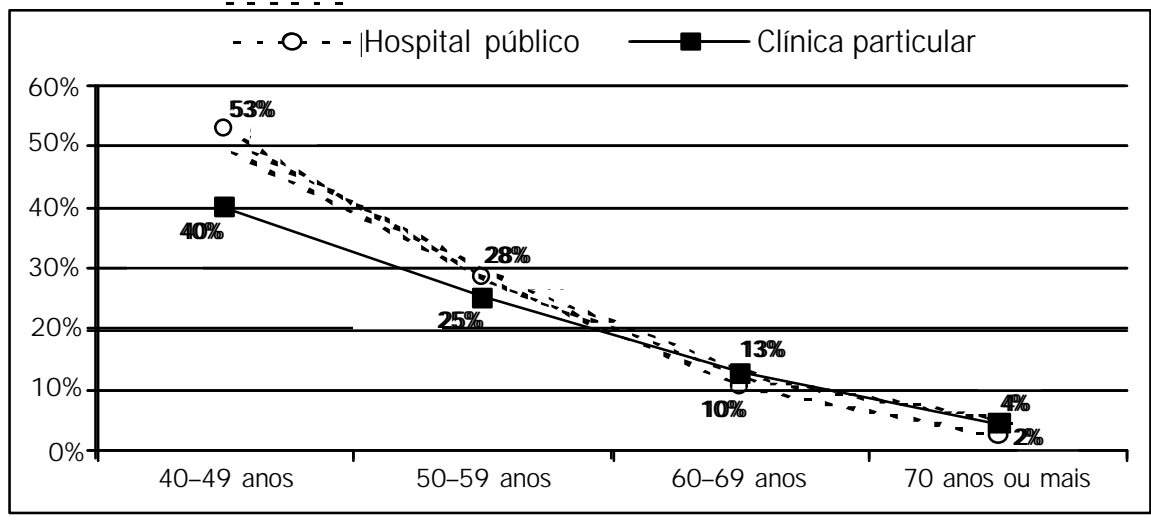

Figura 3. Hospital público e clínica privada. Redução da proporção de mulheres examinadas com o avançar da idade.

Aproximadamente $10 \%$, neste mesmo local, referiram dificuldade financeira como razão para não terem se submetido ao estudo radiográfico mamário prévio. Na clínica privada, $9 \%$ das mulheres disseram não terem sido submetidas à mamografia antes porque não haviam se consultado. Estes dados revelam que cerca de $80 \%$ dos exames, de ambos os centros investigados, não são executados por falha de condução do processo de rastreamento do câncer de mama por parte do médico assistente. Uma em cada três mulheres pesquisadas no hospital público já havia se consultado anteriormente e apesar de o exame ter sido solicitado, não o executaram, provavelmente por falta de orientação adequada quanto à sua importância. Este achado é condizente com a descrição de Vieira e $\operatorname{Koch}^{(31)}$,em que apenas $5,8 \%$ das mulheres que já tinham realizado mamografia anteriormente afirmaram terem sido recomendadas pelos seus médicos. A simples condição de fornecer o pedido para realização do exame não parece ser suficiente para engajar a mulher no processo de detecção precoce do câncer mamário.

Do exposto até aqui, constata-se a necessidade premente de inserção do médico assistente na questão do rastreamento do câncer de mama, sob pena de os recursos de um programa visando à detecção precoce desta doença sofrerem canalização para destino equivocado, conforme constataram

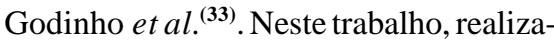
do em hospital de ensino, os autores verificaram que 50,4\% das mulheres submetidas a mamografia naquela instituição tinham idade inferior a 35 anos, e, destas,
$73 \%$ eram assintomáticas. Apenas $7 \%$ daqueles exames eram realizados com a intenção de rastreamento do câncer de mama.

Verificação interessante foi a de que "medo do exame" foi razão praticamente desprezível para as mulheres que nunca tinham se submetido a mamografia.

Em média, as mulheres da clínica privada tinham 1,7 mamografia anterior e as do serviço público, 2,1. A última mamografia tinha sido realizada, em média, há 2,4 anos no primeiro caso e há 1,7 ano no segundo. De acordo com Kerlikowske et $a l .{ }^{(16)}$, a sensibilidade da mamografia é afetada pelo intervalo de tempo entre os exames, sendo a menor sensibilidade identificada quando se adotam intervalos de 25 meses. Salientam, ainda, que examinar a população feminina mais jovem a cada dois anos pode resultar em maior número de cânceres intervalares do que a avaliação mamográfica anual. A casuística mostra tendência a alargamento do intervalo entre os exames, indicando que o exame pode estar sendo utilizado em sua faixa de menor sensibilidade.

Entre $20 \%$ e $30 \%$ das entrevistadas estavam em TRH, e destas, aproximadamente um quinto não tinha mamografia anterior. Kavanagh et al. ${ }^{(34)}$ relatam que a sensibilidade da mamografia diminui cerca de 12,5\% em usuárias de TRH, evidenciandose a importância de acompanhamento estreito dessas mulheres ${ }^{(35,36)}$. Apesar das controvérsias, existem fortes evidências de que existe elevação do risco de câncer de mama nas usuárias de TRH. As neoplasias nas mulheres em TRH se comportam de forma mais indolente, com doença mais confinada e maior sobrevida, devido à maior vigilância a que estão sujeitas essas

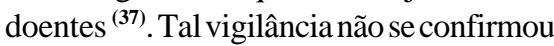
em parcela significativa das participantes do estudo.

A falta de uma postura cautelosa quanto ao uso da TRHé mais preocupante ainda nas mulheres com história familiar de câncer de mama. Neste grupo existe o somatório de fatores de risco ${ }^{(38,39)}$, porém verifica-se que nenhum cuidado especial foi dispensado a elas. A casuística revelou que $21,05 \%$ das usuárias de TRH do hospital público tinham antecedentes familiares de câncer de mama, sem terem sido submetidas a mamografia prévia. Na clínica privada, $4 \%$ das entrevistadas não tinham mamografia anterior. História familiar de câncer de mama foi descrita por $7,5 \%$ e $13,3 \%$ das analisadas na clínica privada e no hospital público, respectivamente. Destas, 33,3\% não tinham mamografia anterior no primeiro caso, e 28,7\%, no segundo. As médias de idade foram de 45 anos e 47 anos, na mesma ordem. Estas mulheres possuem risco relativo de desenvolver câncer de mama duas a três vezes maior que o restante da população femini$\mathrm{na}^{(\mathbf{5 , 4 0 , 4 1 )}}$. Apesar disso, não se identifica uma preocupação em controlá-las de forma criteriosa.

Retirada de nódulo foi a causa mais comum de intervenção mamária no hospital público. Já na clínica privada, retirada de nódulo, punção e cirurgia plástica responderam, cada um, por cerca de $30 \%$ das intervenções mamárias. Ressalte-se aqui a elevada proporção de retirada de nódulos $(27,3 \%)$ e mastectomias $(50 \%)$ às cegas, sem análise mamográfica preliminar. A literatura é clara quanto aos benefícios da mamografia antes da mastectomia, auxiliando no planejamento da cirurgia e proporcionando intervenções mais limitadas, com evidentes benefícios social, funcional e humano ${ }^{(42-44)}$.

Dentro das motivações para a realização da mamografia, o rastreamento respondeu por aproximadamente dois terços dos exames nas entrevistadas com idade superior a 35 anos. Em segundo lugar (cerca de $15 \%$ ) situou-se a investigação de mastalgia. Nas mulheres com menos de 35 anos, este sintoma foi responsável por $35 \%$ e $44 \%$ das solicitações de mamografia no 
hospital público e na clínica particular, respectivamente. Marconato e Boff ${ }^{(29)}$ questionam o contraste entre o hábito de se solicitar mamografia devido à mastalgia e a freqüência da associação deste sintoma com o câncer.

Em ambos os serviços cerca de 11,5\% das mulheres não tinham amamentado, enquanto tempo de amamentação superior a seis meses foi referido por cerca de dois terços das entrevistadas. Apesar de ser fonte de polêmica, a amamentação é aceita como fator protetor contra o câncer de mama. Tessaro et al. ${ }^{(45)}$ conduziram estudo em 172 casos de câncer de mama, com grupo controle, não comprovando este efeito, porém os próprios autores sugerem, ao final do trabalho, novas investigações sobre o tema.

Desafortunadamente, os resultados revelam uma realidade complexa. O médico parece ser um dos maiores responsáveis pela condução ineficaz do processo de rastreamento do câncer de mama. Na outra ponta do problema está a desinformação da mulher. Responderam que não têm o hábito de se consultar regularmente $9 \%$ das entrevistadas no serviço particular, e que não fizeram o exame solicitado por o considerarem desnecessário $30 \%$ das entrevistadas no hospital público. Após a justificativa de não ter feito a mamografia por falta de pedido médico, estes foram os outros motivos mais representativos. Os três totalizaram $94,5 \%$ das justificativas da clínica privada e 79,5\% do hospital público. A falta do hábito de se consultar e a rotulação de desnecessário ao exame solicitado são fruto de falha no processo de orientação da paciente por parte do médico assistente, que não tem valorizado ou explorado o lado educacional do ato médico. Partilhamos do posicionamento de Koch e Peixoto(13): "Para a resolução do problema é necessária atitude de enfrentamento da situação, que requer altos investimentos em educação e pesquisa. O processo educacional deve ser dirigido tanto aos profissionais de saúde como à população em geral, visando, especialmente, ao diagnóstico precoce e à prevenção."

Por fim, os resultados demonstram que a elaboração e implantação de um programa de detecção precoce do câncer de mama deverá, imperiosamente, incluir estratégia para inserção e conscientização da classe médica no problema, sob pena de os investimentos em infra-estrutura não encontrarem onde ecoar.

\section{CONCLUSÕES}

As mulheres têm chegado para o primeiro exame mamográfico com idade média de 49 anos.

O grupo etário mais examinado foi de 40-49 anos.

Houve queda acentuada do número de mulheres submetidas a mamografia a partir dos 50 anos de idade.

O grupo etário dos 35-39 anos representou parcela mínima das examinadas no hospital público, podendo constituir uma tendência a não se realizar mamografia de base.

Houve sobrecarga do hospital público com mulheres de outras cidades ou estados.

Cerca de $40 \%$ das mulheres com mais de 40 anos não possuíam mamografia anterior.

TRH sem avaliação mamográfica prévia foi identificada em aproximadamente $20 \%$ das entrevistadas.

Das mulheres com história familiar de câncer de mama, cerca de $30 \%$ nunca tinham se submetido a mamografia

Procedimento intervencionista, sem avaliação mamográfica preliminar, foi freqüente.

A principal motivação clínica para realização de mamografia foi o rastreamento do câncer de mama.

A segunda maior indicação de mamografia foi a investigação de mastalgia.

Falta de solicitação médica de mamografia foi a principal justificativa para mulheres em idade de rastreamento do câncer de mama não terem realizado o exame.

Cerca de $30 \%$ das entrevistadas no hospital público não tinham realizado mamografia anteriormente, por a julgarem desnecessária.

Medo do exame não representou fator de impedimento à realização do exame.

\section{REFERÊNCIAS}

1. Brasil. Ministério da Saúde. Sistema Único de Saúde. Datasus. Sistema de informações de mortalidade. Disponível no endereço eletrônico: http: //www.datasus.gov.br/cgi/idb2000/d05f.html Acessado em: 10/6/2001.

2. Brasil. Ministério da Saúde. Instituto Nacional do Câncer. Pró-Onco. Estimativa da incidência e mortalidade por câncer de mama no Brasil. Disponível no endereço eletrônico: http://www.inca. org.br/epidemiologia/estimativa2001/index.html. Acessado em 10/6/2001.

3. Wun LM, Feuer EJ, Miller BA. Are increases in mammographic screening still a valid explanation for trends in breast cancer in the United States? Cancer Causes Control 1995;6:135-44.

4. Almeida OJ, Zeferino JC, Teixeira LC. Mamografia de rastreamento em mulheres idosas. Femina 1999;27:627-9.

5. Barton MB, Harris R, Fletcher SW. Esta paciente tem câncer de mama? O exame clínico de triagem da mama: deve ser feito? como? JAMA Brasil 2000;4:2641-61.

6. Baségio DL, Koch HA. Formas de diagnóstico do câncer de mama na mulher gaúcha. Rev Bras Mastol 1998;8:64-71.

7. Baségio DL. Métodos de diagnóstico do câncer de mama - uma contribuição a "Bases para um programa de detecção precoce do câncer de mama”. [Tese de Doutorado]. Rio de Janeiro: Universidade Federal do Rio de Janeiro, 1999.

8. Berg JW. Clinical implications of risk factors for breast cancer. Cancer 1984;53(3 Suppl):589-91.

9. Easton DF, Bishop DT, Ford D, Crockford GP. Genetic linkage analysis in familial breast and ovarian cancer: results from 214 families. The Breast Cancer Linkage Consortium. Am J Hum Genet 1993;52:678-701.

10. Hulka BS, Stark AT. Breast cancer: cause and prevention. Lancet 1995;346:883-7.

11. Tavassoli FA. General considerations. In: Tavassoli FA, ed. Pathology of the breast. 2nd ed. Hong Kong: McGraw-Hill, 1999:27-74.

12. Debs YD, Sánchez FB, Carvalho CCD, Araújo SS. Aspectos atuais da prevenção primária do câncer de mama. Rev Bras Mastol 2000;10:22-7.

13. Koch HA, Peixoto JE. Bases para um programa de detecção precoce do câncer de mama por meio da mamografia. Radiol Bras 1998;31:329-37.

14. Santos RP, Koch H, Frasson A, Schenato A, Melo GS. Diagnóstico precoce do câncer de mama: o papel do carcinoma ductal in situ. Rev Bras Mastol 1998;8:25-9.

15. Mendonça MHS. Análise crítica dos métodos de imagem na detecção e diagnóstico do câncer mamário. Radiol Bras 1999;32:289-300.

16. Kerlikowske K, Grady D, Barclay J, Sickles EA, Ernster V. Effect of age, breast density, and family history on the sensitivity of first screening mammography. JAMA 1996;276:33-8.

17. Baker LH. Breast Cancer Detection Demonstration Project: five-year summary report. CA Cancer J Clin 1982;32:194-225.

18. Eiras AL, Koch HA, Peixoto JE. Parâmetros envolvidos na qualidade da imagem mamográfica - revisão dos fundamentos teóricos. Rev Imagem 2000;22:143-8

19. Kopans DB. The breast cancer screening controversy: lessons to be learned. J Surg Oncol 1998; 67:143-50.

20. Kopans DB. Updated results of the trials of screening mammography. Surg Oncol Clin N Am 1997;6:233-63.

21. Sickles EA. Breast cancer screening outcomes in women ages 40-49: clinical experience with service screening using modern mammography. J Natl Cancer Inst Monogr 1997;(22):99-104.

22. Sickles EA, Kopans DB. Deficiencies in the analysis of breast cancer screening data. J Natl Cancer Inst 1993;85:1621-4. 
23. Michaelson JS, Halpern E, Kopans DB. Breast cancer: computer simulation method for estimating optimal intervals for screening. Radiology 1999;212:551-60.

24. Ferreira RS, Peixoto JE, Koch HA, Drexler G. Mamografias de alta resolução: uma avaliação do risco e do benefício. Radiol Bras 1998;31:2216.

25. Pasqualete HA. Prevenção secundária do câncer de mama. In: Pasqualete HA, Koch HA, SoaresPereira PM, Kemp C. Mamografia atual. Rio de Janeiro: Revinter, 1998:89-97.

26. Brasil. Ministério do Planejamento e Orçamento. Instituto Brasileiro de Geografia e Estatística. Diretoria de Pesquisa. Departamento de população e indicadores sociais. População residente censo 2000: Brasil, unidades da federação e municípios. Disponível na internet: http://www.ibge. gov.br. Acessado em 10/6/2001.

27. Brasil. Ministério do Planejamento e Orçamento. Instituto Brasileiro de Geografia e Estatística. IBGE-Cidades@. Disponível na internet: http:// www.ibge.gov.br. Acessado em 23/9/2001.

28. Koch HA, Peixoto JE, Neves ALE. Análise da infra-estrutura para a mamografia no Brasil. Radiol Bras 2000;33:23-9.

29. Marconato M, Boff RA. Importância da mamografia como método de "screening" do câncer de mama na comunidade. Rev Cient AMECS 1993 $2: 125-8$.
30. Santos CC, Pinto Neto AM. Acurácia da mamografia no diagnóstico do câncer de mama inicial em mulheres com idade entre 35 e 49 anos. J Bras Ginecol 1997;107:353-9.

31. Vieira AV, Koch HA. Conhecimento sobre mamografia por mulheres que freqüentam o Serviço de Radiologia da Santa Casa de Misericórdia do Rio de Janeiro (SCMRJ). Rev Bras Mastol 1999;9:56-67.

32. Lamas JM, Pereira MG, Koch HA. Avaliação dos resultados de exames mamográficos para detecção precoce de câncer de mama em mulheres assintomáticas, no Distrito Federal. Radiol Bras 2000;33:161-7.

33. Godinho E, Ximenes CA, Freitas Jr R. Mamografia: o uso do método no Hospital das Clínicas da UFG. J Bras Ginecol 1994;104:269-72.

34. Kavanagh AM, Mitchell H, Giles GG. Hormonereplacement therapy and accuracy of mammographic screening. Lancet 2000;355:270-4.

35. Gondin G, Crippa CG, Traebert EE, et al. Terapia de reposição hormonal e mamografia. Rev Bras Mastol 2001;11:30-3.

36. Finotti MCCF, Freitas Júnior R. A importância da mamografia no climatério. Femina 1998;26: 487-91.

37. Montoro AF. Terapêutica de reposição hormonal e riscos de câncer mamário. (Editorial). Rev Bras Mastol 2000;10:1-2.

38. De Luca LA, Zambotti RP, Tobias P, Uemura G,
Schimitt. Terapêutica de reposição hormonal e câncer de mama. Rev Bras Mastol 1998;8:4251.

39. Pascalicchio JC, Fristachi CE, Baracat FF. Câncer de mama: fatores de risco, prognósticos e preditivos. Rev Bras Mastol 2001;11:71-84.

40. Souza RM, Defferrari R, Lazzaron AR, Scherer L, Borba AA, Frasson AL. Relação da história familiar em primeiro grau com câncer de mama. Rev Bras Mastol 1998;8:123-8.

41. Giglio AD, Bendit I, Barros A. Aconselhamento de mulheres com riscos hereditários de câncer de mama: um guia para o mastologista. Rev Bras Mastol 2000;10:138-47.

42. Perry N. Fundamento del screening por mamografia: evidencia de sua eficacia. Rev Argent Radiol 1995;59:209-10.

43. Melo ALKO, Meneses ACO, Scandiuzzi D, Oliveira MK, Chaud TL. Detecção e prognóstico do câncer "precoce" de mama: revisão da literatura e apresentação de 12 casos. Radiol Bras 2000;33: 279-85.

44. Crespo TM, Galbán AT. Valor de la mamografia en el diagnóstico del cáncer de mama. Acta Cancerol 1995;25:126-8.

45. Tessaro S, Béria JU, Tomasi E, Zerwes DP, Becker JD, Vieira C. Amamentação e câncer de mama na zona sul do Rio Grande do Sul: um estudo controle. Rev Bras Mastol 1997;7:155-62. 